Military Technical College

Kobry Elkobbah,

Cairo, Egypt

May 27-29,2008

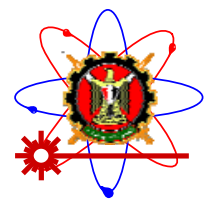

$4^{\text {th }}$ International Conference on Mathematics and Engineering Physics (ICMEP-4)

EM-1

\title{
MODELING OF A PASSIVE SCALAR IN A TURBULENT FREE JET UNDER THE CORIOLIS EFFECT HASNAOUI ${ }^{*} \mathrm{M}$.
}

\begin{abstract}
The influence of Coriolis forces is studied in stationary and $S_{c}=1$ (Schmidt number) cases on the tran sport of a passive scalar in a three-dimensional inhomogeneous turbulent free jet. The mean velocity field defines a small parameter $U_{\infty}$ (non-dimensional wind-speed). This parameter governs the asymptotic expansion of the analysis. Using a linear spectral approach, two-point velocity-concentration correlation equations are obtained under some realistic simplifying assumptions. The solution depends on the non-uniform mean concentration gradient. Taking into account swirl characteristics, an expansion of the solution is constructed by means of analytical tools, including the effects of several fundamental physical parameters. The mean concentration gradient field possesses a privileged direction. As application of the model, the spectral components of the solution are determined using further simplifying approximations. Graphical surfaces are drawn: these surfaces show the specific characteristics of mixing in turbulent jets. The results are matched with other results obtained by means of numerical methods and experimental results.
\end{abstract}

\section{KEY WORDS}

Inhomogeneous turbulence, Coriolis forces, Linear spectral approach, Asymptotic analysis.

\footnotetext{
* Department of structures and materials, Air Royal School, Marrakech (Morocco)
} 


\section{INTRODUCTION}

The prediction of the passive scalar dispersion in a turbulent flow is an interesting problem linked to the importance in the diffusion of heat and mass transfers and the environment problems. Its determination, in a space, is based on the knowledge of the flow around the emission source.

Often in engineering problems, the particular objective involves obtaining comparatively rapid rates of mixing, and for this reason practical problems generally focus on scalar mixing in turbulent flows.

In the turbulent mixing of a passive scalar, surfaces of constant mixed-fluid concentration, or isoscalar surfaces, are highly convoluted in turbulent-jet flows, as well as other high-Reynolds-number turbulent flows. Knowledge of the geometry of these isosurfaces is necessary for an understanding of the turbulent mixing process. In particular, molecular mixing occurs across such surfaces [1].

A key quantity in the mixing and transport process is the turbulent scalar flux $\overline{\hat{u}_{i} \hat{c}}$, with $\hat{u}_{i}$ and $\hat{c}$ are the velocity and the scalar fluctuations, respectively. It is the quantity that is accounting for the effects of turbulence on the mean scalar profile. In order to characterize the contributions of the different turbulent length scales to the scalar flux, it is important to analyze the spectral distribution of scalar flux over wave numbers, i.e., the scalar flux spectrum. In the literature, the spectral distribution of scalar flux produced by a uniform scalar gradient imposed on isotropic turbulence was extensively studied [2, 3, 4].

One question that can be asked is whether theories that have been successful in describing the behavior of the turbulent energy can be applied to the dynamics of a passive scalar with a mean gradient. Many theories have been used to describe a variety of turbulent systems. For example, the Eddy Damped Quasi-Normal Markovian theory (EDQNM), including the classical studies of the energy and isotropic scalar spectrum [5,6,7], to more exotic problems involving mean flow inhomogeneities [8]. Recently, a model based on two-point closure theory of turbulence is proposed and applied to study the Reynolds number dependency of the scalar flux spectra in homogeneous shear flow with a cross-stream uniform scalar gradient [9]. The evolution of a passive scalar in a turbulent flow is characterized by scalar and velocity gradients, whose statistical correlations have clear physical meanings.

We are interested, in this paper, in the diffusion and the transport of a passive scalar of a three-dimensional turbulent inhomogeneous free jet in the presence of a mean concentration gradient submitted to Coriolis forces. Because of its "pathological behavior", the interaction of Coriolis effects with the turbulence originated an active research several years ago. This question arises in various problems encountered in engineering science [10] and geophysical applications [11]. Recently, the statistical correlations between the passive scalar gradient and vorticity in homogeneous and isotropic turbulence submitted to a solid body rotation, is studied by Gence [12].

The problem is described by a linear spectral approach. Such an approach appears as less empirical than most usual models and allows to separately taking into account the various scales of turbulence. It also allows to simply eliminating a problem related to the pressure, and the turbulent diffusion due to triple correlations in velocities and velocity-velocity concentration-correlation is neglected. In this view, we assume that there exists a particular direction along which the jet develops. This circumstance occurs, for instance, if the jet is emitted from a source (for instance the orifice of a chimney). In this model, there exists a small parameter $U_{\infty}$ characterizing 
the non-dimensional wind-speed. This parameter governs the asymptotic analysis. The use of both combined spectral analysis and asymptotic tools then allows considering practical problems: for instance, a good understanding of the influence of Coriolis forces on a turbulent structure may improve our ability to predict the rotating flows.

In what follows, for simplicity, the jet is assumed directed towards the east. In Sect.2, firstly, the results of the spectral tensor of a model involving a free three-dimensional and inhomogeneous jet are briefly exposed. Secondly, the one-point correlation velocity-concentration equations are first formulated under some assumptions and Boussinesq approximation. These equations are adimensiona1 and they are applied in stationary and Schmidt number $\mathrm{Sc}=1$. These equations are then rewritten in spectral form using a Fourier analysis, in order to define the spectral correlations of our model. Combining both spectral analysis and asymptotic analysis, the solutions of these equations are determinate. In sect 3, some examples are considered, for which we estimate the application ranges of this model.

\section{FORMULATION OF PROBLEM AND RESOLUTION}

We consider a three-dimensional inhomogeneous turbulent free jet. The jet is emitted from a point-source in an atmospheric medium in the presence of gravity and Coriolis forces. The fluid, moving in terrestrial frame, is incompressible and viscous. The flow is assumed stationary. The equations of motion are the Navier-Stokes equations with Boussinesq approximation, the energy equation and the passive scalar transport equation.

At large Reynolds number, we obtain [13, 14] the adimensional equations of the spectral tensor $\Phi$ (obtained by three-dimensional Fourier transforms of the two point velocities correlations), namely:

$$
\begin{gathered}
\frac{\partial \Phi_{k j}}{\partial t}+\bar{u}_{m} \frac{\partial \Phi_{k j}}{\partial x_{m}}+2 k_{\perp}^{2} \Phi_{k j}+\left[\Phi_{m j} \frac{\partial \bar{u}_{k}}{\partial x_{m}}+\Phi_{k m} \frac{\partial \bar{u}_{j}}{\partial x_{m}}\right]-\frac{2}{k_{\perp}^{2}} \frac{\partial M}{\partial x_{m}}\left[k_{k} \Phi_{m j}+k_{j} \Phi_{k m}\right] \\
-\frac{R_{f}}{\sigma_{T}} \Phi_{3 j} \frac{\partial \bar{u}_{3}}{\partial x_{3}} \delta_{3 k}+\frac{2}{k_{\perp}^{2}} \delta_{1 k}\left[\frac{\partial M}{\partial x_{2}}\left(k_{1} \Phi_{2 j}+k_{j} \Phi_{12}\right)+\frac{\partial M}{\partial x_{3}} k_{1} \Phi_{3 j}\right] \\
=-\varepsilon\left[\Phi_{k 1}\left[\frac{\partial \bar{u}_{3}}{\partial x_{1}} \delta_{3 j}+2 \frac{\partial \bar{u}_{2}}{\partial x_{1}} \delta_{2 j}\right]+\frac{1}{R_{o}}\left(\varepsilon_{2 l j} \omega_{2} \Phi_{k l}+2 \varepsilon_{3 l j} \omega_{3} \Phi_{2 l} \delta_{2 k}\right)\right. \\
\left.+\frac{1}{k_{\perp}^{2}}\left\{\left[\frac{k_{2}-k_{3} / t g}{R_{o}}-2 \frac{\partial M}{\partial x_{1}}\right]\left(k_{k} \Phi_{1 j}+k_{j} \Phi_{k 1}-\delta_{1 k} k_{1} \Phi_{1 j}\right)\right\}\right] .
\end{gathered}
$$

where we have set:

$$
\omega_{1}=0 ; \omega_{2}=1 / \operatorname{tg} \varphi ; \omega_{3}=1 \text { and } \mathrm{k}=1,2,3 ; \mathrm{m}=2,3
$$

In which $\bar{u}_{k i}$ denotes the components of mean velocity field; $\mathrm{k}_{1}, \mathrm{k}_{2}$ and $\mathrm{k}_{3}$ are the components of the wave vector $\vec{k}$ along $\mathrm{x}_{1}, \mathrm{x}_{2}$ and $\mathrm{x}_{3}$ directions, respectively. $k_{\perp}^{2}\left(=k_{2}^{2}+k_{3}^{2}\right)$ is the transverse wave number, and $M=k_{j} \bar{u}_{j}$ measures the interaction between the wave vector and mean velocity field. $R_{o}=u_{o} / \Omega L$ is the Rossby number ( $\mathrm{L}$ is the horizontal length scale, $u_{o}$ is a characteristic speed of the turbulent fluctuations) and $\omega_{j}$ are the components of the Coriolis forces. $\delta_{i j}$ is the symmetric 
Kronecker symbol. $R_{f}=G_{R} \mathrm{~N}^{2} /\left(\frac{\partial \bar{u}_{3}}{\partial x_{3}}\right)^{2}$ is the flux Richardson number associated with the shear of the mean flow (where $G_{R}$ is the Grashof number, and $N$ is the BruntVàisâlà frequency of the ambient medium). $\sigma_{T}$ is the turbulent Prandtl number and usually it is approximately chosen equal to 0.9 [15]. The small parameter $\varepsilon=d / L$ ( $\mathrm{d}$ is the thickness of the jet) characterises the relative thickness of this layer, and this parameter allows to construct a boundary layer approximation as well as to provide additional equations necessary for the analysis.

Moreover, the Eq.(1) is valid under the asymptotic restriction:

$$
\varepsilon=R_{e}^{-1} \quad \text { and } \quad U_{\infty} \prec \prec 1
$$

relating the Reynolds number of the flow and the stratification of the medium. The relation (3) seems to be restrictive, but, from a mathematical point of view, the first characterizes a phenomenon where the stratification and dissipation originate competing influences and the second characterizes the regularity condition of these equations.

For a case of analytical solution, it is assumed that $\Phi_{k j}$ 's admits the following asymptotic expansions:

$$
\Phi_{k j}=\Phi_{k j}^{o}+\varepsilon \Phi_{k j}^{1}+\varepsilon^{2} \Phi_{k j}^{2}+\cdots
$$

The general form of this equation may be written as:

$$
L_{k j}(\Phi)=-\varepsilon A_{k j}
$$

where $L_{k j}(\Phi)$ denotes the linear operator and $A_{k j}$ are the secular terms.

By prescribing that $\varepsilon \Phi_{k j}^{1}$ is uniformly small before $\Phi_{k j}^{0}$ in Eq (1), and cancelling the secular terms $\left(A_{k j}=0\right)$, we obtain, at order 0 , these equations:

$$
L_{k j}\left(\Phi_{k j}^{0}\right)=0
$$

At large Reynolds number, and using scaled variables, the non-dimensional equations for the velocity-concentration correlations, read:

$$
\begin{aligned}
\bar{u}_{k} \frac{\partial \overline{\hat{u}_{i} \hat{c}}}{\partial x_{k}}+ & +\left[\overline{\hat{u}_{i} \hat{u}_{m}} \frac{\partial \bar{c}}{\partial x_{m}}+\overline{\hat{u}_{m} \hat{c}} \frac{\partial \bar{u}_{i}}{\partial x_{m}}\right]+\delta_{1 i}\left(\overline{\hat{u_{1}} \hat{c}} \frac{\partial \bar{u}_{1}}{\partial x_{1}}-\overline{\hat{u}_{3} \hat{c}} \frac{\partial \bar{u}_{1}}{\partial x_{3}}\right) \\
& -\hat{u}_{i} \frac{\partial^{2} \hat{c}}{\partial x_{m} \partial x_{m}}-S_{c} \hat{c} \frac{\partial^{2} \hat{u}_{i}}{\partial x_{m} \partial x_{m}}+\hat{c} \frac{\partial \hat{\mathrm{p}}}{\partial x_{i}}-\delta_{1 i} \hat{c} \frac{\partial \hat{\mathrm{p}}}{\partial x_{1}}=0
\end{aligned}
$$

where $\bar{c}$ is the mean concentration and $\hat{p}$ is the pressure fluctuation.In the sequel, we are going to study the closure of the equation (7). 


\subsection{Linear spectral analysis}

We now introduce the two-point correlations of the velocity-concentration $\overline{\hat{u}_{i}^{\prime} \hat{c}^{\prime \prime}}$. These correlations are defined using two points nearby $x$, say $x^{\prime}$ and $x^{\prime \prime}$, in the following manner:

$$
\hat{u}_{k}^{\prime}=\hat{u}_{k}\left(x^{\prime}\right), \hat{c}^{\prime \prime}=\hat{c}\left(x^{\prime \prime}\right), r=x^{\prime \prime}-x^{\prime}, r_{k}=x^{\prime \prime}{ }_{k}-x^{\prime}{ }_{k}, x=\left(x^{\prime}+x^{\prime \prime}\right) / 2
$$

By using the procedure previously applied to the one-point correlations, we obtain, for the two-point correlations, and after some calculation, the equation satisfied by these correlations, namely:

$$
\begin{gathered}
\bar{u}_{k} \frac{\partial \overline{\hat{u}_{i}{ }_{i} \hat{c}^{\prime \prime}}}{\partial x_{k}}+\left[\overline{\hat{u}_{i}^{\prime} \hat{u}^{\prime \prime}{ }_{m}} \frac{\partial \bar{c}}{\partial x_{m}}+\overline{\hat{u}_{m}^{\prime} \hat{c}^{\prime \prime}} \frac{\partial \bar{u}_{i}}{\partial x_{m}}\right]+\delta_{1 i}\left(\overline{\hat{u}_{1}^{\prime} \hat{c}^{\prime \prime}} \frac{\partial \bar{u}_{1}}{\partial x_{1}}-\overline{\hat{u}^{\prime}{ }_{3} \hat{c}^{\prime \prime}} \frac{\partial \bar{u}_{1}}{\partial x_{3}}\right) \\
-\left(1+S_{c}\right) \frac{\partial^{2} \overline{\hat{u}_{i}{ }_{i} \hat{c}^{\prime \prime}}}{\partial r_{m} \partial r_{m}}+\frac{\partial \overline{\hat{p}^{\prime} \hat{c}^{\prime \prime}}}{\partial x_{i}}-\delta_{1 i} \frac{\partial \overline{\hat{p}^{\prime} \hat{c}^{\prime \prime}}}{\partial x_{1}}=0
\end{gathered}
$$

The corresponding equations for the two-point pressure-concentration correlation can be rewritten after some computation in the form:

$$
\frac{\partial^{2}}{\partial r_{m} \partial r_{m}}\left(\overline{\hat{p}^{\prime} \hat{c}^{\prime \prime}}\right)=2 \frac{\partial \bar{u}_{k}}{\partial x_{m}} \frac{\partial \overline{\left(\overline{\left.\hat{u}_{m}^{\prime} \hat{c}^{\prime \prime}\right)}\right.}}{\partial r_{k}}
$$

Note, that the correlation $\overline{\hat{u}_{i}{ }_{i} \hat{u}_{m}^{\prime \prime}}$ is the two-point Reynolds stress.

In order to convert the correlation equations into spectral forms, we now introduce three-dimensional Fourier transforms of the variables figuring in (9) and (10) with respect to $r$ in the following forms:

$$
\left\{\begin{array}{l}
\overline{\hat{u}_{\mathrm{i}}^{\prime} \hat{u}^{\prime \prime}}{ }_{j}=\int_{-\infty}^{+\infty} \Phi_{i j}^{o}\left(x_{1}, x_{2}, x_{3}, \vec{k}\right) e^{I \vec{k} \cdot \vec{r}} d k_{1} d k_{2} d k_{3} \\
\overline{\hat{u}_{\mathrm{i}}^{\prime} \hat{c}^{\prime \prime}}=\int_{-\infty}^{+\infty} F_{i c}\left(x_{1}, x_{2}, x_{3}, \vec{k}\right) e^{I \vec{k} \cdot \vec{r}} d k_{1} d k_{2} d k_{3}
\end{array}\right.
$$

where $I=(-1)^{1 / 2}$ is the complex number. So, the Fourier transforms take the simplified forms:

$$
\begin{gathered}
\bar{u}_{k} \frac{\partial F_{i c}}{\partial x_{k}}+\Phi_{i m}^{o} \frac{\partial \overline{\mathrm{c}}}{\partial x_{m}}+F_{m c} \frac{\partial \bar{u}_{i}}{\partial x_{m}}+\delta_{i 1}\left(F_{1 c} \frac{\partial \bar{u}_{1}}{\partial x_{1}}-F_{3 c} \frac{\partial \bar{u}_{1}}{\partial x_{3}}\right) \\
-\left(1+S_{c}\right) k_{\perp}^{2} F_{i c}-\frac{I}{k_{2}}\left[F_{3 c} \frac{\partial^{2} \bar{u}_{2}}{\partial x_{i} \partial x_{3}}+\frac{\partial F_{3 c}}{\partial x_{i}} \frac{\partial \bar{u}_{2}}{\partial x_{3}}\right. \\
\left.-\delta_{i 1} \frac{\partial \bar{u}_{2}}{\partial x_{3}} \frac{\partial F_{3 c}}{\partial x_{1}}\right]=0
\end{gathered}
$$

The different terms of the equation (12) express the contributions of various processes to the rate of change of the spectral velocity-concentration correlations. 


\subsection{Asymptotic analysis}

Before we solve Eqs (12), it is of interest to expand the spectral tensor $\Phi_{i j}^{o}$, the mean concentration $\bar{c}$ and the mean velocity $\overrightarrow{\vec{u}}$ with respect to $U_{\infty}$. In this view, we assume that these functions admit the following asymptotic expansions:

$$
\begin{aligned}
& \Phi_{i j}^{o}=\Phi_{i j(0)}^{o}+U_{\infty} \Phi_{i j(1)}^{o}+\ldots . . \\
& \bar{c}=\bar{c}_{0}+U_{\infty} \bar{c}_{1}+\left(U_{\infty}\right)^{2} \bar{c}_{2}+\ldots . . \\
& \bar{u}_{i}=\bar{u}_{i}^{o}+U_{\infty} \bar{u}_{i}^{1}+\left(U_{\infty}\right)^{2} \bar{u}_{i}^{2}+\ldots . .
\end{aligned}
$$

Returning now to Eqs (12), the general form of this equation may be written as:

$$
\left(F_{i c}\right)=U_{\infty} H_{i c}
$$

where $\mathfrak{I}$ is the linear operator, namely:

$$
\mathfrak{I}\left(F_{i c}\right)=\left(\bar{u}_{m}^{o} \frac{\partial}{\partial x_{m}}-2 k_{\perp}^{2}\right) F_{i c}+\Phi_{i m(0)}^{o} \frac{\partial \bar{c}_{0}}{\partial x_{m}}
$$

and $H_{i c}$ is the secular term, namely:

$$
\begin{aligned}
H_{i c}= & \bar{u}_{k}^{1} \frac{\partial F_{i c}}{\partial x_{k}}+F_{i c}+\Phi_{\mathrm{im}(\mathrm{o})}^{\mathrm{o}} \frac{\partial \overline{\mathrm{c}}_{1}}{\partial x_{m}} \\
& -\frac{I \delta_{i 3}}{k_{2}}\left[F_{3 c} \frac{\partial^{2} \bar{u}_{2}^{1}}{\partial x_{3} \partial x_{3}}+\frac{\partial F_{3 c}}{\partial x_{3}} \frac{\partial \bar{u}_{2}^{1}}{\partial x_{3}}-2 I k_{2} F_{3 c}\right] \\
& +\delta_{i 2}\left[F_{3 c} \frac{\partial \bar{u}_{2}^{1}}{\partial x_{3}}-F_{2 c}-\frac{I}{k_{2}} \frac{\partial F_{3 c}}{\partial x_{3}} \frac{\partial \bar{u}_{2}^{1}}{\partial x_{3}}\right]
\end{aligned}
$$

A solution of Eqs.(16) may now be looked for by using a generalized asymptotic expansion with respect to $U_{\infty}$, namely:

$$
F_{i c}=F_{i c}^{o}+U_{\infty} F_{i c}^{1}+\left(U_{\infty}\right)^{2} F_{i c}^{2}+\ldots \ldots
$$

\subsection{Determination of the spectral correlations}

By prescribing that $U_{\infty} F_{i c}^{1}$ is uniformly small before $F_{i c}^{o}$ in Eq. (16), we obtain the no degenerate equations respectively at order 0 and order 1 with respect to $U_{\infty}$.

We remark at the differential equations level of the $F_{i c}^{o}$ 's and the $F_{i c}^{1}$ 's, that their left hand sides are the same. But, the right hand side of the $F_{i c}^{1}$ 's, exhibits the terms which characterize the secular term. This last term depend on the functions defined at order 0 with respect to $U_{\infty}$. Thus, knowledge of the solutions at order 0 with respect to $U_{\infty}$, allows obtaining the solutions at the following order.

According to the similitude method, the analysis of different scales of differential equations of $F_{i c}^{o}$, allows to write the solutions such: 


$$
F_{i c}^{o}=f\left(\Phi_{i k(0)}^{o}, \partial \bar{c}_{0} / \partial x_{k}\right)
$$

This relation shows that the determination of the $F_{i c}^{o}$ is linked to the determination of the mean scalar gradient $\partial \bar{c}_{0} / \partial x_{i}$. In general, there exists a relationship between the turbulent scalar flux and the mean concentration gradient. Indeed, the studies of turbulent swirling flows [16], in order to establish a prediction procedure and to elucidate the effects of swirl on turbulent transport, are demonstrated that the characteristic features due to swirl could be predicted by this relation kind. Recently, it is proposed $[17,9]$ to introduce a tonsorial form namely:

$$
F_{i c} \propto \Delta_{i j}\left(\partial \bar{c} / \partial x_{j}\right)
$$

in which $\Delta_{i j}$ is quantity that corresponds to components of a tensorial spectral flux. In what follows, for simplicity, we consider the following hypothesis:

$$
\partial \bar{c}_{0} / \partial x_{2}=0
$$

and the only nonzero components of the spectral tensor are in this case $\Phi_{12(0)}^{o}$, $\Phi_{22}^{o}$ and $\Phi_{33}^{o}$. So, taking into account the hypothesis above, the solutions are of the form:

$$
F_{1 c}^{o}=0, \quad F_{2 c}^{o}=\Phi_{12(o)}^{o}\left(\partial \bar{c}_{o} / \partial x_{1}\right), F_{3 c}^{o}=\Phi_{33(o)}^{o}\left(\partial \bar{c}_{o} / \partial x_{3}\right)
$$

these solutions characterizing the generation terms of swirling production.

Because of the presence of the secular terms, the operator $H_{i c}^{o}$ acts, in general, on all components of the spectral correlation $F_{i c}^{o}$. Hence, the procedure for solving (16), we consider the $H_{i c}^{o}=0$ case. Inserting the solutions (23) in Eqs of $F_{i c}^{o}$, we obtain the solutions of the problem.

The spectral analysis allows taking into account the various scales of turbulence. Indeed, integration is made over a sphere whose radius is equal to the modulus of the wave number; accordingly turbulent structures are only characterized by their sizes. Consequently, the spectral components $\Psi_{k j}$ and $N_{i c}^{o}$ are computed with a spherical average operation, namely:

$$
\begin{aligned}
& \Psi_{k j}\left(x_{i}, k\right)=k^{2} \int_{0}^{\pi} \int_{0}^{2 \pi} \Phi_{k j}^{o}\left(x_{i}, k\right) \sin (\theta) \mathrm{d} \alpha \mathrm{d} \theta \\
& N_{i c}^{o}\left(x_{i}, k\right)=k^{2} \int_{0}^{\pi} \int_{0}^{2 \pi} F_{i c}^{o}\left(x_{i}, k\right) \sin (\theta) \mathrm{d} \alpha \mathrm{d} \theta
\end{aligned}
$$

where $\theta$ is angle between $\vec{k}$ and $\mathrm{x}_{3}(\mathrm{z})$-axis; $\alpha$ is angle between $\vec{k}$ and $\mathrm{x}_{1}(\mathrm{x})$-axis. These spectral correlations are influenced by the Coriolis force and by the various physical factors such as: atmospheric conditions (stability and instability), convection phenomenon, the geometrical characteristic of the chimney, the speed of the jet at the exit, etc. 


\section{APPLICATIONS}

In order to validate the preceding results, we now present some examples for which we estimate the application ranges of these models. In these examples, the adimensional velocity $U_{\infty}$ is defined as: $0.1 \leq U_{\infty} \leq 0.2$ and the adimensional speed at the exit of the chimney $W_{J}$ (it represents the bulk of evacuated smoke) is defined as: $0.8 \leq W_{J} \leq 1$. The latitude is fixed: $\varphi=\pi / 6$. A vortex size $\eta$ measured by a correlation is therefore associated to a wave number $k$ such: $k \eta=0(1)$. A vortex will be then a perturbation the energy of which would be concentrated at vicinity of wave number $k$. But, in physical space, the vortex appears as an object with fuzzy contours. So, the large structure corresponds to large $\eta$ (small $k$ ), while the small structure corresponds to small $\eta$ (large k). Also, the Coriolis force is characterized by the Rossby number $R_{0}$. So, the weak rotation corresponds to $R_{0} » 1$ case and the large rotation corresponds to $\mathrm{R}_{\mathrm{o}}$ «1.

Figure (1) shows the influence of the Coriolis forces on the coherent structures. Approximate singularities in the flow appear in the plane of the density spectral $\Psi_{12}$ since, slender cones pointing towards the location of the big structures. The intensity of these structures decreases when the rotation increases [18, 19, 20]. It is now recognized that the wavelengths (phase, amplitudes) correlated to vortical structures within a single realization determine many properties of the turbulence.

Figure (2, left) illustrates that the large structures associated to the mixing are not influenced by the Coriolis force. Indeed, we observe that the mean concentration gradient has a privileged direction parallel to the development axis $(x \geq 0)$ of the jet, and its intensity is uniform. Figure (2, right) illustrates the effect of the Coriolis force on the small structures. When the rotation rate is increased, the intensity of the mean concentration gradient increases along the development axis and the altitude. Also, it changes the sign. In particular at large Rossby number the direction of the scalar gradient tends to become parallel to the $z$ axis.

Figure (3) illustrates the influence of the geometrical characteristics of the chimney on the subsequent development of the initial perturbation. The intensity of $N_{2 c}^{o}$ decreases along the jet axis. When the rotation increases, we observe the appearance of the coherent vortices "longitudinal hairpin vortex", elongated along $x$ direction, reconnecting the Kelvin-Helmholtz instabilities [21]. The spectral correlation $N_{2 c}^{o}$ involving the correlation axisymmetric with respect to the axis pointing in the direction of the mean gradient. When the rotation increases, we observe a stagnation of the $N_{2 c}^{o}$ at vicinity the singularity $z=0$.

\section{CONCLUSION}

The results obtained in the present paper globally show the importance of the Coriolis forces in the modeling of the transport and dispersion of a passive scalar in inhomogeneous turbulence, and the power of the spectral approach for this modeling. The several characteristics usually encountered in the inhomogeneous turbulence are correctly predicted by our model.

When this model is applied to the study of the appearance of coherent structures associated to a passive scalar field and to the influence of the Coriolis forces on their evolution, the matching of predictions and numerical simulations with our model remains quantitatively acceptable. Moreover, they allow confirming much experimental behavior. 


\section{REFERNCES}

[1]. Catrakis, J. H. and Dimotakis, P. E., 1. Fluid Mech, 317, 369-406 (1996).

[2]. Mydlarski, L., "Mixed velocity-passive scalar statistics in high-Reynolds number turbulence," J. Fluid Mech. 475, 173 (2003).

[3]. O'Gorman, P. A. and Pullin, D. I., "Effect of Schmidt number on the velocityscalar cospectrum in isotropic turbulence with a mean scalar gradient,"J. Fluid Mech. 532, 111 (2005).

[4]. Bos, W. J. T., Touil, H. and Bertoglio, J. P., "Reynolds number dependency of the scalar flux spectrum in isotropic turbulence with a uniform scalar gradient," Phys.

Fluids 17, 125108 (2005).

[5]. Orszag, S.A., "Analytical theories of turbulence". J. Fluid Mech., Vol. 41, p. 363 (1970).

[6]. Lesieur, M. and Herring, J.R., ' Diffusion of a passive scalar in two-dimensional turbulence“. J. Fluid Mech., 161, pp. 77-95 (1985).

[7]. Lesieur, M., Comte; P. and Metais, O., "Numerical simulations of coherent vortices in turbulence“. Appl. Mech. Rev., 48, n³, 121-149 (1995).

[8]. Nakauchi, N. and Sega, S., "The homogeneous axisymmetric passive scalar in isotropic turbulence". Phys. Fluids., Vol. 30, 337 (1987).

[9]. Bos, W. J. T. and Bertoglio, J.P., "Inertial range scaling of scalar flux spectra in uniformly sheared turbulence“.Phys. Fluids 19, 025104 (2007).

[10]. Khellaf, K. and Lauriat, G., " Heat transfer in vertical rotating annuli. A numerical study“. Rev. Gen. Thermique, 34,104-116 (1995).

[11]. Cho, J.Y.K. and Polvani, L., 'The emergence of jets and vortices in freely evolving shallow water turbulence on a sphere ". Phys. FI., 8, 1531-1552 (1996). [12]. Gence; J.N., "Couplage statistique entre vorticité et gradient de scalaire passif dans une turbulence statistiquement homogène et isotrope mise en rotation". C. R. Mécanique. 335, pp. 93-98 (2007).

[13]. M. Hasnaoui, M. and Agouzoul, M., "'Linear spectral analysis of threedimensional inhomogeneous turbulent free jet under realistic atmospheric conditions". AMSE Periodical Journal.Modelling B, vol. 71, no.5, pp. 1-22 (2002). [14]. M. Hasnaoui, M. and Agouzoul, M., "Determination of the inhomogeneous initial spectrum in a turbulent jet emitted from a point source". Phys. Chem. News, vol. 30, pp. $72-775$ (2006).

[15]. Launder, B.E., Reece, G.J. and Rodi, W., "Progress in the development of a Reynolds-stress turbulence closure ". J. of Fluid Mech., vol. 68, part 3, pp. 537-566 (1975).

[16]. Hirai, S. and Takagi, T., "'Parameters Dominating Swirl Effects on Turbulent Transport Derived from Stress-Scalar-Flux Transport Equation “. Int.J.Heat Mass Transfer. 38, N.12, 2175-2182 (1995).

[17]. Bos, W. J. T., Touil, H., Shao, L. and Bertoglio, J.-P., "On the behaviour of the velocity-scalar cross correlation spectrum in the inertial range," Phys. Fluids 16, 3818 (2004).

[18]. Bonnet, J. P., Delville, J., Glauser, M. N., Antonia, R. A., Bisset, D. K., Cole, D. R., Fiedler, H. E., Garem, J. H., Hilberg, D., Jeong, J., Kevlahan, N. K. R., Ukeiley, L. S., Vincendeau, E., " Collaborative testing of eddy structure identification methods in free turbulent shear flows". Experiments in Fluids, vol. 25, pp. 197-225 (1998). [19]. Lesieur, M., Bégou, P., Briand, E., Danet, A., Delcayre, F. and Aider, J.L., "Coherent vortex dynamics in large eddy simulations of turbulence". J. Turbulence, vol. 4, pp. 016 (2003). 
[20]. Cuypers, Y., Maurel, A. and Petitjeans, P., “'Comparison between an experimental turbulent vortex and the lundgren vortex ". J. Turbulence, vol.5, pp. 030 (2004).

[21]. Bonnet, J.P. and Glauser, M.N., “Eddy Structure Identification in Free Turbulent Shear Flows". Kluwer Academic Publishers, Netherlands (1993).

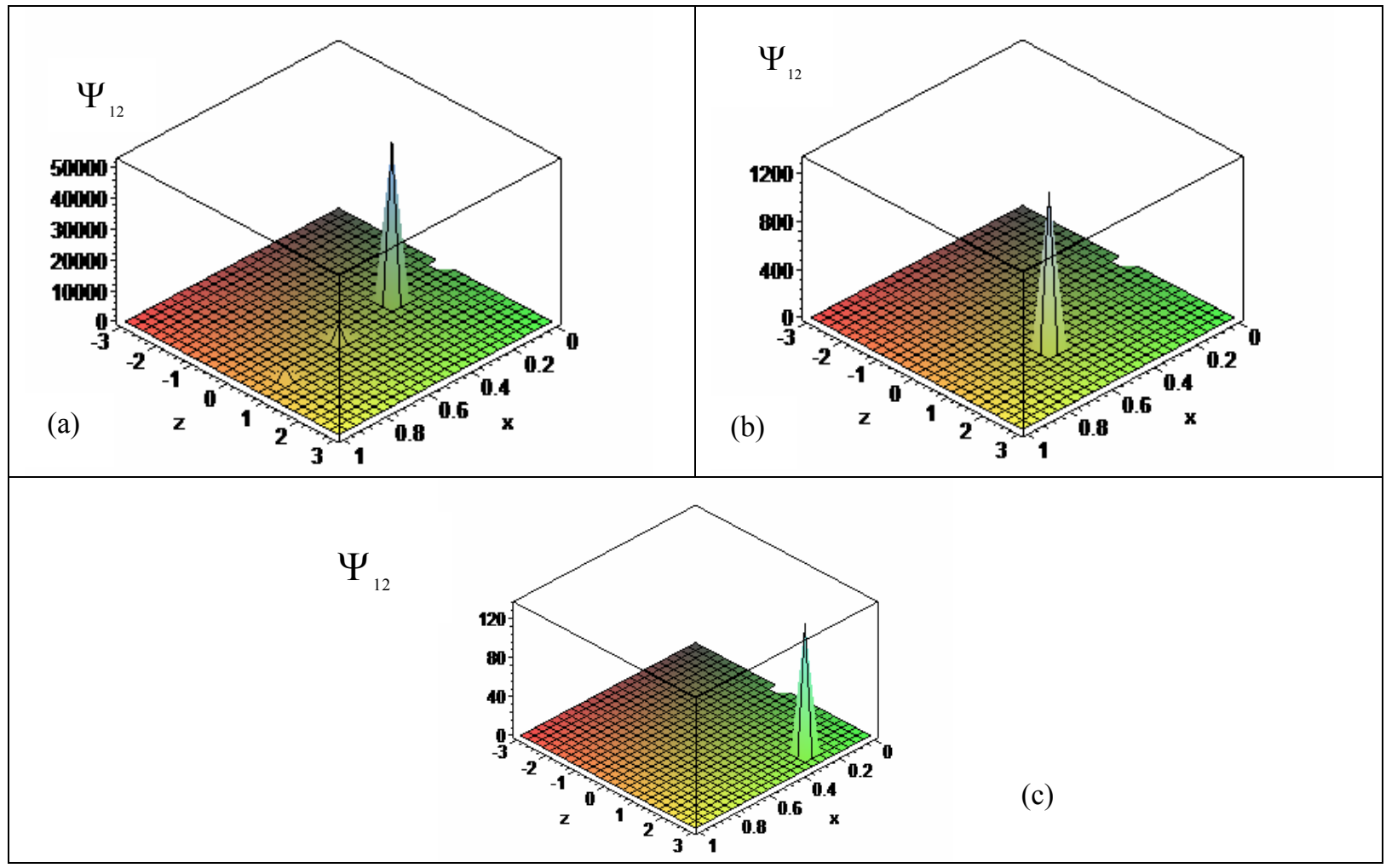

Figure.1. Density spectral $\Psi_{12} \cdot \mathbf{k}=1 / 50 . U_{\infty}=0.1, W_{J}=1, h=100, \varphi=\pi / 6 . x \in[0,1]$ and $z \in[-3,3]$. a) $\mathbf{R o}=\mathbf{2 0}, b) \mathrm{Ro}=\mathbf{8}$, c) $\mathrm{Ro}=\mathbf{2}$.

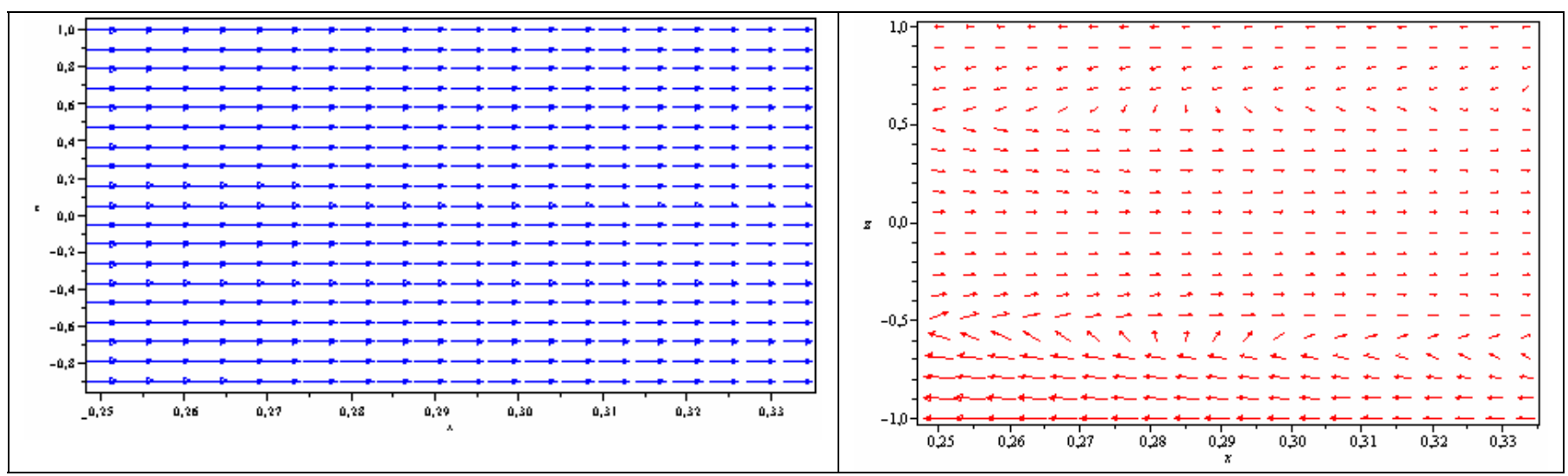

Figure.2. Gradient scalar $\vec{\nabla} \bar{c}_{0} . \mathrm{W}_{\mathrm{J}}=0.9, \mathrm{~h}=100, \varphi=\pi / 6 . \mathrm{x} \in[1 / 4,1 / 3]$ and $\mathrm{z} \in[-1,1] . \mathrm{Ro}=0.1$. Left: $\mathrm{k}_{2}=1 / 100, \mathrm{k}_{3}=1 / 300$. Right: $\mathrm{k}_{2}=1, \mathrm{k}_{3}=1 / 3$. 


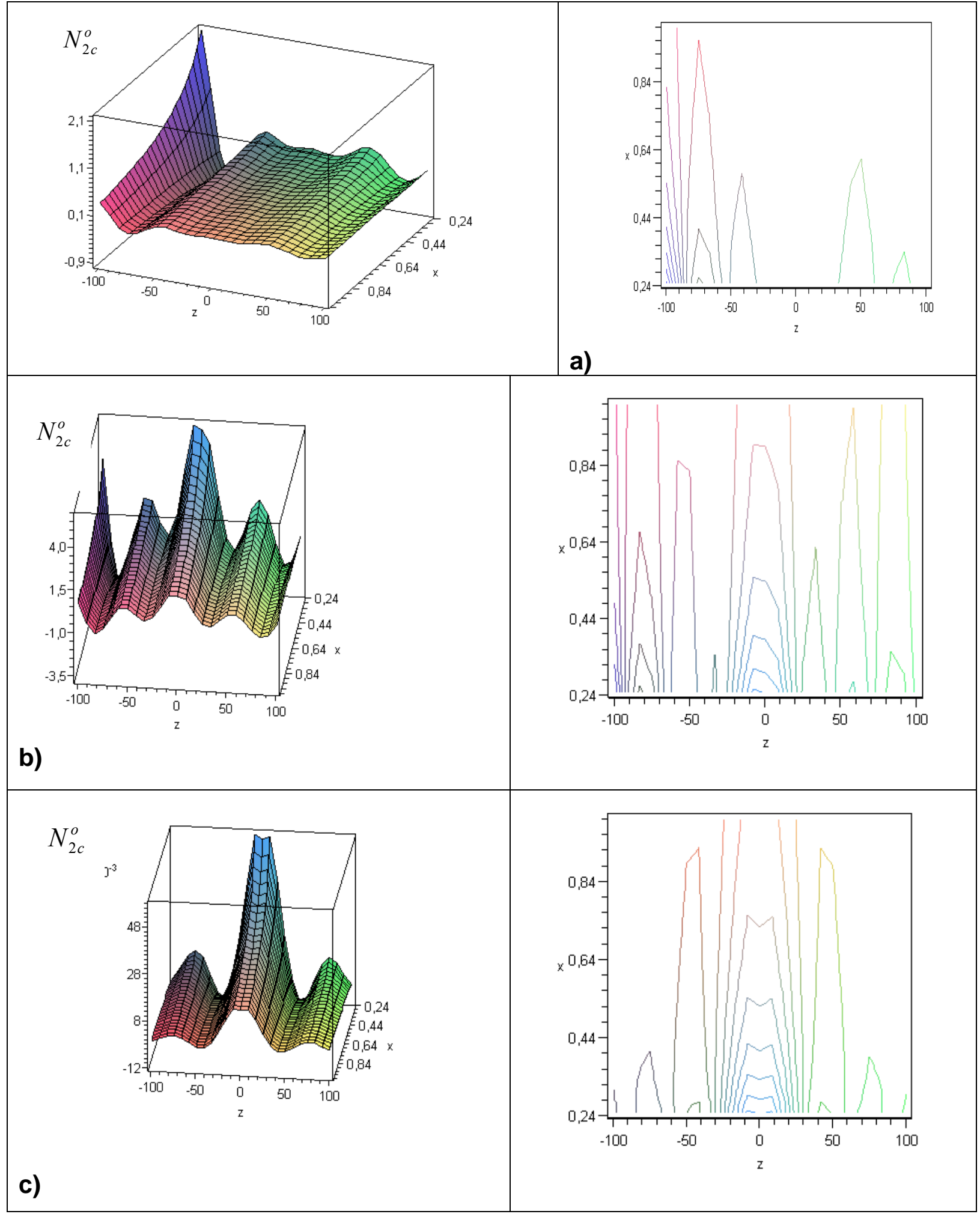

Figure.3. Scalar flux spectrum $N_{2 c}^{o} . \mathrm{k}=1 / 30 . \mathrm{W}_{\mathrm{J}}=0.9, \mathrm{~h}=100, \varphi=\pi / 6 . \mathrm{x} \in[1 / 4,1]$ and $\mathrm{z} \in[-$ 100, 100]. a) $\mathrm{Ro}=100, \mathrm{~b}) \mathrm{Ro}=10$, c) $\mathrm{Ro}=0.1$. 